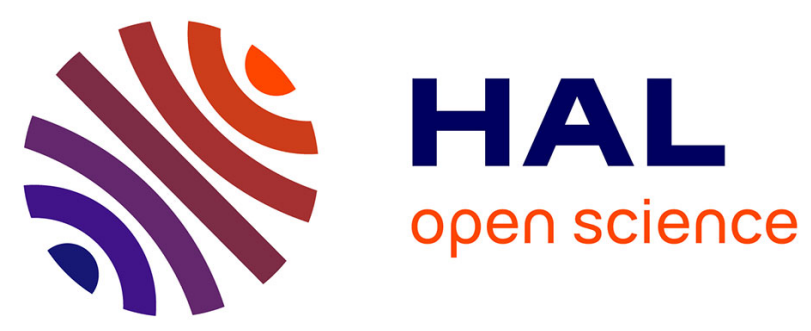

\title{
Interfacial Control of Magnetic Properties at LaMnO3/LaNiO3 Interfaces
}

Marta Gibert, Michel Viret, A. Torres-Pardo, C S Piamonteze, P. Zubko, N. Jaouen, J.-M. Tonnerre, A Mougin, J Fowlie, S. Catalano, et al.

\section{- To cite this version:}

Marta Gibert, Michel Viret, A. Torres-Pardo, C S Piamonteze, P. Zubko, et al.. Interfacial Control of Magnetic Properties at LaMnO3/LaNiO3 Interfaces. Nano Letters, 2015, 15 (11), pp.7355 - 7361. 10.1021/acs.nanolett.5b02720 . cea-01498302

\section{HAL Id: cea-01498302 https://hal-cea.archives-ouvertes.fr/cea-01498302}

Submitted on 29 Mar 2017

HAL is a multi-disciplinary open access archive for the deposit and dissemination of scientific research documents, whether they are published or not. The documents may come from teaching and research institutions in France or abroad, or from public or private research centers.
L'archive ouverte pluridisciplinaire HAL, est destinée au dépôt et à la diffusion de documents scientifiques de niveau recherche, publiés ou non, émanant des établissements d'enseignement et de recherche français ou étrangers, des laboratoires publics ou privés. 


\title{
Interfacial Control of Magnetic Properties at $\mathrm{LaMnO}_{3} / \mathrm{LaNiO}_{3}$ Interfaces
}

\author{
M. Gibert, ${ }^{*}{ }^{\dagger}$ M. Viret, ${ }^{\dagger}+$ A. Torres-Pardo, ${ }^{\S}$ C. Piamonteze, $\|$ P. Zubko, ${ }^{\dagger}$ N. Jaouen, ${ }^{\perp}$ J.-M. Tonnerre, $^{\#}$ \\ A. Mougin, ${ }^{\S}$ J. Fowlie, ${ }^{\dagger}$ S. Catalano, ${ }^{\dagger}$ A. Gloter, ${ }^{\S}$ O. Stéphan, ${ }^{\S}$ and J.-M. Triscone ${ }^{\dagger}$ \\ ${ }^{\dagger}$ Département de Physique de la Matière Quantique, University of Geneva, Geneva, Switzerland \\ ${ }^{\ddagger}$ Service de Physique de l'Etat Condensé, CNRS URA 2464, CEA Saclay, Gif-sur-Yvette, France \\ ${ }^{\S}$ Laboratoire de Physique des Solides, University of Paris-Sud, CNRS-UMR 8502, Orsay 91405, France \\ "Swiss Light Source, Paul Scherrer Institute, 5232 Villigen-PSI, Switzerland \\ ${ }^{\perp}$ Synchrotron SOLEIL, 91192 Gif-Sur-Yvette, France

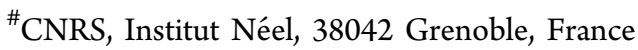

Supporting Information

ABSTRACT: The functional properties of oxide heterostructures ultimately rely on how the electronic and structural mismatches occurring at interfaces are accommodated by the chosen materials combination. We discuss here $\mathrm{LaMnO}_{3} /$ $\mathrm{LaNiO}_{3}$ heterostructures, which display an intrinsic interface structural asymmetry depending on the growth sequence. Using a variety of synchrotron-based techniques, we show that the degree of intermixing at the monolayer scale allows interface-driven properties such as charge transfer and the induced magnetic moment in the nickelate layer to be controlled. Further, our results demonstrate that the magnetic state of strained $\mathrm{LaMnO}_{3}$ thin films dramatically depends on interface reconstructions.

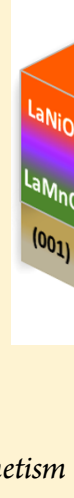

modified by such structural asymmetries. ${ }^{8-10}$ In this letter, we demonstrate that it is possible to completely modify an interfacial property by changing the degree of material intermixing at the monolayer scale. To this end, we build a unique interface in a $\mathrm{LaMnO}_{3} / \mathrm{LaNiO}_{3}(\mathrm{LMO} / \mathrm{LNO})$ bilayer. In order to vary the intermixing at the monolayer level, we use the intrinsic asymmetry of the system arising from the growth sequence. Indeed, we show here that depositing LNO on LMO does not produce the same interface as LMO on LNO. We take advantage of this asymmetry to explore to what extent interfacial structural characteristics influence the resulting physical properties of the system, even though the same materials combination is considered. Using a variety of state-ofthe-art polarization-dependent absorption techniques, we demonstrate that the nature of the LMO magnetic order can be controlled by the degree of asymmetry and intermixing of the interfaces while maintaining perfect lattice coherency. Moreover, the net magnetic moment induced in interfacial

Received: July 9, 2015

Revised: September 27, 2015

Published: October 20, 2015 
(a)

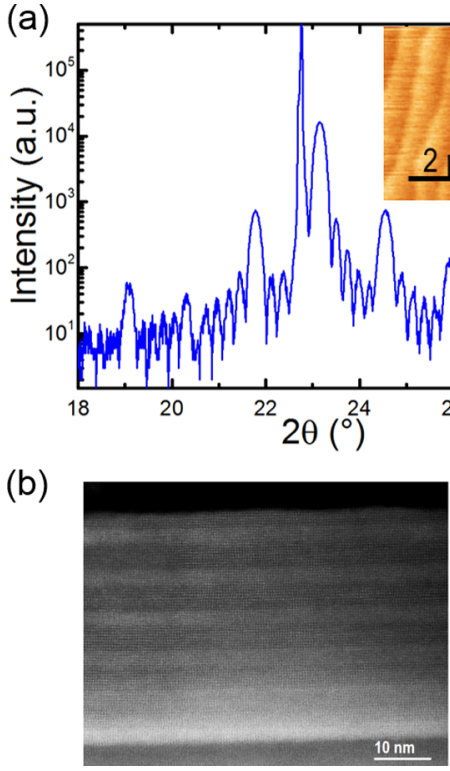

(c)

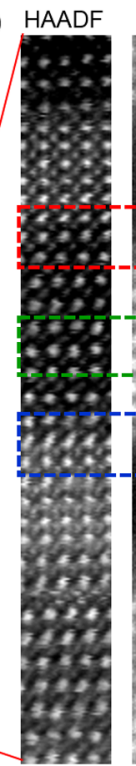

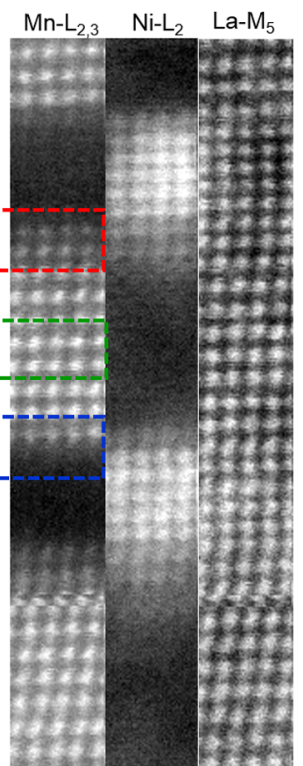
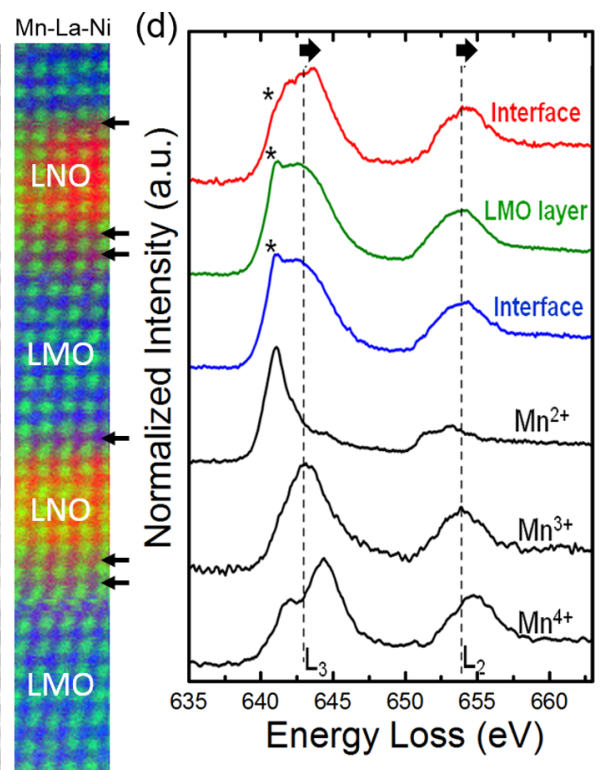

Figure 1. (a) XRD scan for a (8LNO/8LMO) 6 superlattice grown on (001)STO. Inset: atomic force microcopy image of the same sample. (b) Corresponding low magnification HAADF-STEM image. (c) Atomically resolved EELS measurements showing the interfacial structural asymmetry (indicated by arrows) that depends on the growth sequence; growth direction is from bottom to top. (d) EELS spectra of the Mn $\mathrm{L}_{2,3}$-edges for the rough interface (red), the inner LMO layer (green), and the sharp interface (blue). Reference $\mathrm{Mn} \mathrm{L}_{2,3}$-edges for $\mathrm{Mn}^{2+}, \mathrm{Mn}^{3+}$, and $\mathrm{Mn}^{4+}$ are added for comparison (black). ${ }^{33}$ All measurements reveal a $\mathrm{Mn}^{2+}$ signal as a result of beam damage during the scan (marked by asterisk).

LNO layers relies on the charge transfer as determined by the level of intermixing.

The parent compound of the manganites family, LMO, is a Mott insulator with A-type antiferromagnetism $\left(T_{\text {Néel }} \approx 140 \mathrm{~K}\right)$ resulting from cooperative Jahn-Teller distortions, which lead to anisotropic $\mathrm{Mn}-\mathrm{O}-\mathrm{Mn}$ superexchange, i.e., ferromagnetic in-plane and antiferromagnetic out-of-plane. ${ }^{11,12}$ Superimposed to these structural distortions, the rotation of $\mathrm{MnO}_{6}$ octahedra leads to a small canted ferromagnetic moment as a consequence of the Dzialoshinski-Moriya interaction. ${ }^{13}$ These properties occur for bulk stoichiometric orthorhombic LMO $(a=5.54 \AA$, $b=5.72 \AA$, and $c=7.70 \AA$ at room temperature) ${ }^{14}$ Like all manganite compounds, LMO is highly sensitive to doping and structural distortions. When grown as a thin film on cubic $\mathrm{SrTiO}_{3}$ (STO) substrates $(a=3.905 \AA)$, LMO typically displays an insulating ferromagnetic behavior, possibly attributed to off-stoichiometry, cubic in-plane strain imposed by the substrate, development of a specific orbital order, and/or electronic reconstructions due to polar discontinuity. ${ }^{15-19}$ However, the least distorted member of the perovskite nickelates, LNO (rhombohedral structure with a pseudocubic lattice parameter $a_{\mathrm{pc}}=3.84 \AA$ ), is the only member of the family that in bulk is a paramagnetic metal at all temperatures. $^{20-22} \mathrm{~A}$ thickness-driven metal-to-insulator transition however occurs as the LNO film thickness is reduced to just a few monolayers. ${ }^{23,24} \mathrm{LMO} / \mathrm{LNO}$ heterostructures are of interest because of recent observation of exchange bias at low temperature and induced magnetism in LNO. ${ }^{25-29}$ Charge transfer from $\mathrm{Mn}$ to $\mathrm{Ni}$ is an expected interfacial reconstruction driven by the difference in electronegativity. ${ }^{25-28,30,31}$ The resulting $\mathrm{Ni}^{2+}-\mathrm{Mn}^{4+}$ cations are then anticipated to interact ferromagnetically according to Goodenough-Kanamori rules, ${ }^{32}$ like in the case of the insulating ferromagnetic doubleperovskite $\mathrm{La}_{2} \mathrm{NiMnO}_{6}$.

LNO/LMO superlattices with periodicity ( $N-\mathrm{LNO} / \mathrm{M}$ LMO) $)_{x}$, where $N$ and $M$ indicate the number of unit cells (u.c.) of LNO and LMO, respectively, and $x$, the period repetitions, were grown by off-axis radiofrequency magnetron sputtering on (001)-oriented $\mathrm{TiO}_{2}$-terminated STO substrates. ${ }^{25}$ Figure 1a shows a typical X-ray diffraction (XRD) scan of a LNO/LMO heterostructure where well-defined superlattice peaks and thickness fringes are distinguished in agreement with the designed structure period. The samples display smooth topographies characterized by atomically flat terraces separated by 1 u.c. high steps (inset Figure 1a). Atomically resolved high-angle annular dark field scanning transmission electron microscopy (HAADF-STEM) images of the superlattice projected onto the (010) plane confirm the coherent and epitaxial growth of the heterostructure and the absence of secondary phases (Figure $1 \mathrm{~b}$, and HAADF in Figure 1c). In the case of HAADF-STEM images, the intensity scales roughly with the value of $Z^{\sim 1.7}$. Therefore, the brighter features correspond to the position of the heavier elements, the lanthanum atoms $(Z=57)$ in both the LNO and LMO layers, while the weaker spots in between show nickel $(Z=28)$ and manganese $(Z=25)$ atom columns whose intensities are similar. Spatially resolved electron energy loss spectroscopy (EELS) elemental maps corresponding to the $\mathrm{La}-\mathrm{M}_{5}, \mathrm{Mn}-\mathrm{L}_{2,3}$ and $\mathrm{Ni}-\mathrm{L}_{2}$ edges reveal a structural asymmetry between interfaces depending on the growth sequence (Figure 1c): the growth of LMO-on-LNO displays an atomically flat interface, whereas the LNO-on-LMO interface is diffuse with about 2-3 monolayers roughness. As exemplified in Figure 1d, the EELS spectra at $\mathrm{Mn}_{2,3}$ edges shift to higher energies close to the rough interface compared to the inner LMO layers or the abrupt interface, indicating an enhancement of the $\mathrm{Mn}$ oxidation state in this $2-3$ u.c. intermixed region (see also Supporting Information (SI)). ${ }^{34}$ This increase of the Mn formal valence is attributed to electron transfer from $\mathrm{Mn}$ to $\mathrm{Ni}$ leading to the configuration $\mathrm{Ni}^{2+}-\mathrm{Mn}^{4+}$. A lowering of the branching ratio $I\left(\mathrm{~L}_{3}\right) /\left[I\left(\mathrm{~L}_{3}\right)+I\left(\mathrm{~L}_{2}\right)\right]$ is also observed at the rough interface, consistent with a larger $\mathrm{Mn}^{4+} / \mathrm{Mn}^{3+}$ ratio (SI). ${ }^{35}$ 
(a)

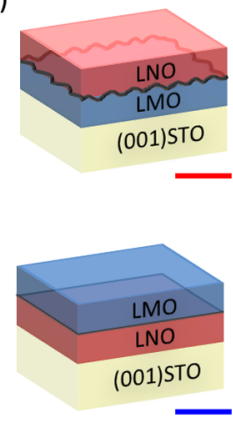

(b)

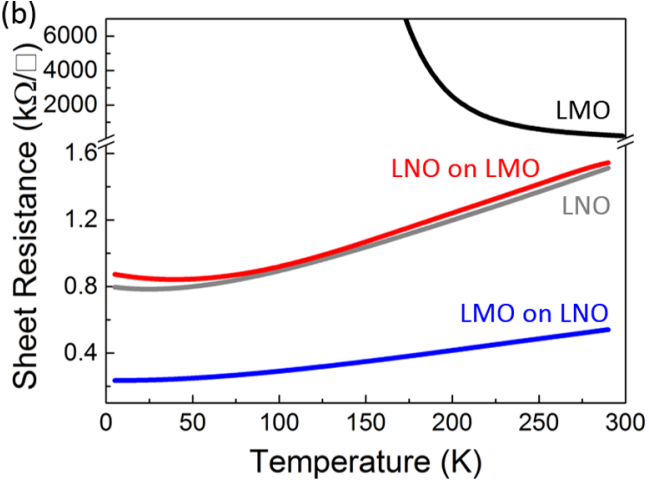

(c)

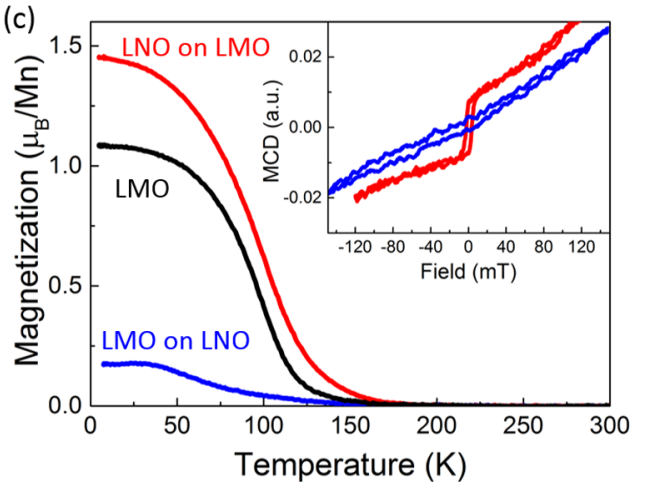

Figure 2. (a) Schematics of the LMO-LNO bilayers investigated, i.e., the rough-interface (LNO/LMO) $1 / /(001)$ STO (top) and the abruptinterface $(\mathrm{LMO} / \mathrm{LNO})_{1} / /(001) \mathrm{STO}$ (bottom) sample. (b) Sheet resistance versus temperature for $(7 \mathrm{LNO} / 7 \mathrm{LMO})_{1} / /(001) \mathrm{STO}$ (red), (7LMO/ $7 \mathrm{LNO})_{1} / /(001)$ STO (blue), and 7LNO//(001)STO (gray). A 28 u.c. LMO film//(001)STO (black) is also shown. (c) Temperature-dependence of the magnetization for two bilayers with $N=M=10$ u.c. and a film $10 \mathrm{LMO} / /(001)$ STO in $0.05 \mathrm{~T}$ with the magnetic field applied parallel to the sample plane. LNO films are paramagnetic. Inset: optical MCD measurements at $90 \mathrm{~K}$ as a function of magnetic field for two bilayers with $N=M=$ 12 u.c. acquired using a polarizer at $45^{\circ}$ to the optical axes of a modulator $(50 \mathrm{kHz})$ and a lock-in detection scheme. In all plots here and throughout this letter, the blue line corresponds to the sharp-interface LMO-on-LNO bilayer and the red line to the intermixed-interface LNO-on-LMO one.

Therefore, there is an intrinsic structural asymmetry between the two LMO-LNO interfaces with a concomitant asymmetry in charge transfer, with both phenomena present throughout the entire superlattice and independent of the periodicity. In oxide heterostructures, it is known that surface segregation and polar discontinuities can be self-limiting processes for interfacial sharpness. ${ }^{8-10}$ In the present study, such asymmetric profiles might be related to surface migration of $\mathrm{Mn}$ triggered by the difference in transition metal ionic radii. The polar discontinuity existing between STO and the LNO or LMO layers is expected to be resolved within the monolayers close to the STO substrate $e^{36,37}$ and is thus unlikely to be the driving-force for the structural modifications observed at the LNO-LMO interfaces.

In order to single out the influence of the structural interface asymmetry on the resulting physical properties of the system, we focus here on bilayers where just one of the two kinds of interfaces is present. Figure 2a sketches the configuration of the samples investigated, i.e., $(N-\mathrm{LNO} / \mathrm{M} \text {-LMO })_{1} / /(001) \mathrm{STO}$ and $(M-L M O / N-L N O)_{1} / /(001) S T O$. Unless otherwise stated, in the examples investigated $N=M$. Further details on sample growth and characterization are provided in the SI. In all cases, XRD reciprocal space maps confirmed the coherent epitaxial growth of the heterostructures on the STO substrates. DC transport measurements for two bilayers $N=M=7$ u.c. are shown in Figure $2 \mathrm{~b}$. The sheet resistance of the sharp-interface LMO-on-LNO sample is clearly reduced compared to that of the rough interface bilayer or a 7-u.c.-thick LNO thin film. Given the insulating behavior of LMO when strained to STO substrates, it then appears that the total bilayer conductivity is dominated by LNO. This difference in conductance of the two bilayers is attributed to the specific growth sequence. Polar and/or oxygen octahedral distortions occurring on the free surface of LNO are known to deteriorate the metallic conductivity of the nickelate films. ${ }^{38}$ The effect of capping the LNO layers with LMO (= sharp interface configuration) suppresses such distortions and improves conductivity (blue vs gray curve in Figure 2b). Confirming this scenario, the relative increase of conductivity of the capped LNO-layer compared to the equivalently thick noncapped LNO film is found to be more significant for low period bilayers, where the contribution of the upper (surface) distorted layers to the total LNO thickness represents a major proportion (see SI).

Striking differences are also found in the temperature dependence of the magnetization of the bilayers. Figure $2 c$ exemplifies the case of bilayers with $N=M=10$ u.c. together with an epitaxial ferromagnetic 10-u.c.-thick LMO film also grown on (001)STO and shown for comparison. LNO thin films are paramagnetic. The rough interface sample (LNO-onLMO, red curve) displays ferromagnetic behavior similar to the bare LMO film but with enhanced magnetization. More surprisingly, the magnetization of the sharp interface bilayer (LMO-on-LNO, blue curve) is drastically reduced. Such dissimilar magnetic properties are also observed for the other bilayer thicknesses investigated (i.e., $N=M=7$ u.c., 12 u.c., etc.). Other measurements including optical magnetic circular dichroism $^{39}$ (MCD) confirm these magnetic results (inset Figure 2c).

In the absence of interfacial reconstructions, the magnetic properties of both bilayers are expected to be comparable to those of the bare LMO film. In order to explore the origin of these very different magnetic behaviors, we performed a variety of X-ray absorption spectroscopy (XAS) experiments at the XTreme beamline of the Swiss Light Source (SLS), Paul Scherrer Institut (PSI). ${ }^{40}$ Figure $3 a$ presents the $\mathrm{Mn}$ absorption $\mathrm{L}_{2,3}$ edges for bilayers consisting of $N=M=10$ u.c. and a 10 u.c. LMO film acquired at $2 \mathrm{~K}$ in $30^{\circ}$ incidence in total electron yield (TEY) mode after field cooling in $0.05 \mathrm{~T}$ with the field parallel to the sample plane. The XAS spectrum of the rough interface LNO-on-LMO sample is clearly shifted to higher energies confirming the enhanced interfacial charge transfer, i.e., larger $\mathrm{Mn}^{4+} / \mathrm{Mn}^{3+}$ ratio, for this bilayer type consistent with the TEM studies on the superlattices. Interestingly, the coupling between $\mathrm{Ni}^{2+}-\mathrm{Mn}^{4+}$ is expected to be mediated by ferromagnetic superexchange, ${ }^{32}$ which should enhance the magnetization as observed for this rough interface bilayer. Hole-doping of manganites favors double-exchange and thus also ferromagnetism. ${ }^{41}$ However, the double-exchange interaction should also be accompanied by an increase of metallicity, which is not observed for this bilayer configuration, in agreement with TEM analysis showing that $\mathrm{Mn}^{4+}$ is confined to the vicinity of the intermixed interface. The shift of the Mn XAS spectra could also be related to a larger amount of oxygen 

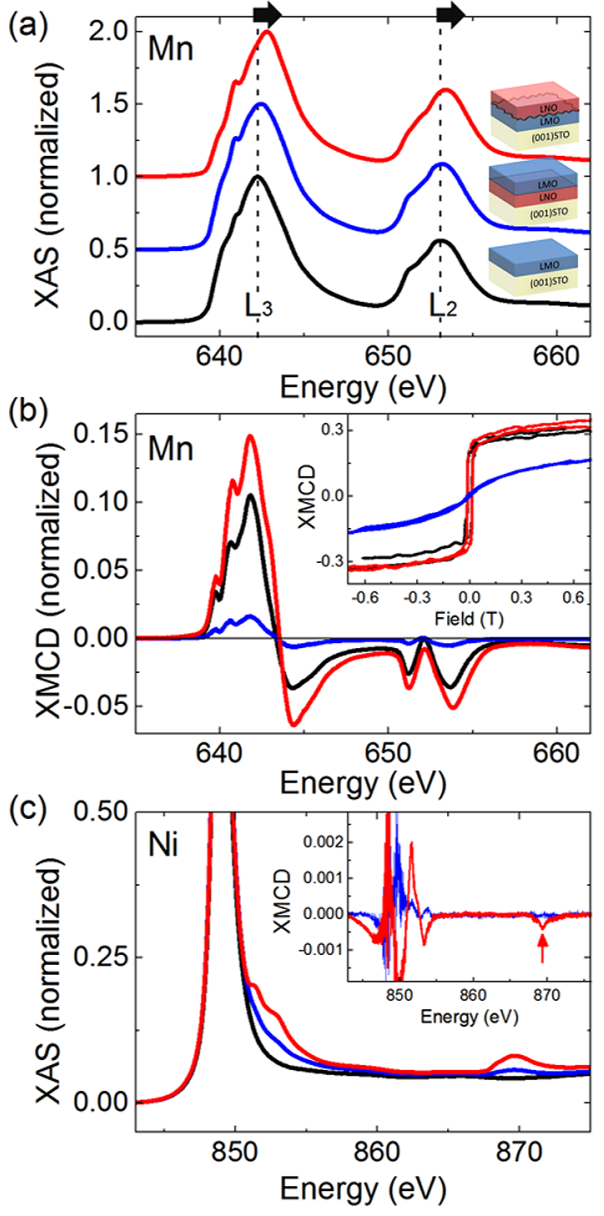

Figure 3. (a) XAS and (b) XMCD measurements at the $\mathrm{Mn}_{2,3}$ edges corresponding to a rough interface $(10 \mathrm{LNO} / 10 \mathrm{LMO})_{1} / /(001) \mathrm{STO}$ (red), a sharp interface (10LMO/10LNO $)_{1} / /(001)$ STO (blue), and a $10 \mathrm{LMO} / /(001)$ STO (black) sample. Inset in (b): XMCD-hysteresis loops at $\mathrm{L}_{3}$ edge for the same set of samples. (c) XAS scans at the $\mathrm{Ni}$ $\mathrm{L}_{2,3}$ edge for the 10 u.c. period bilayers. Notice the overlap of the $\mathrm{Ni}-\mathrm{L}_{3}$ edge and the $\mathrm{La}-\mathrm{M}_{4}$ edge measured on the 10 u.c. LMO film (black line). Inset: corresponding XMCD spectra at $\mathrm{Ni}_{2,3}$ edge, with the arrow indicating the presence of a net magnetic moment on $\mathrm{Ni}$ for the case of the rough interface bilayer (red). The noisy region before the $\mathrm{Ni} \mathrm{L}_{3}$ edge comes from $\mathrm{La} \mathrm{M}_{4}$. All absorption measurements were carried out at $2 \mathrm{~K}$ in $0.05 \mathrm{~T}$ after field cooling in the same field.

vacancies, ${ }^{12}$ but given the same growth conditions and again the reduced conductivity of this sample, this mechanism is unlikely to be dominant. The same features (energy shift of Mn $\mathrm{L}_{2,3}$ edges, reduction of branching ratio) are also observed for a trilayer heterostructure consisting of $10 \mathrm{LNO} / 10 \mathrm{LMO} / 10 \mathrm{LNO}$ (SI), further validating that interfacial charge transfer is at the heart of the enhanced magnetization of the bilayers with a rough interface. However, these results do not disclose the origin of the reduced magnetization of the sharp-interface heterostructure.

$\mathrm{X}$-ray magnetic circular dichroism (XMCD) corroborates the extremely reduced magnetization for the sample with the sharp interface (LMO-on-LNO) observed in SQUID and optical MCD. The XMCD spectra at the $\mathrm{Mn}_{2,3}$ edges at $2 \mathrm{~K}$ in 0.05 $\mathrm{T}$ are presented in Figure $3 \mathrm{~b}$, where the XMCD signal is obtained with plus and minus helicities of circularly polarized light. The XMCD hysteresis curves at the $\mathrm{Mn} \mathrm{L}_{3}$ (inset Figure $3 \mathrm{~b})$ show that the sharp-interface LMO-on-LNO bilayer presents high saturation fields and reduced remanence, a behavior reminiscent of competing antiferromagnetic interactions, in contrast with the square loops displayed by the two other ferromagnetic samples. XAS and XMCD spectra at $\mathrm{Ni}$ $\mathrm{L}_{2,3}$ edges face the problem of energy overlap between the $\mathrm{Ni}$ $\mathrm{L}_{3}$ and $\mathrm{La} \mathrm{M}_{4}$ edges. Nevertheless, and despite the low intensity, magnetic dichroism is unambiguously distinguished at the $\mathrm{Ni} \mathrm{L}_{2}$ edge of the rough interface bilayer revealing the presence of a net magnetic moment in those $\mathrm{Ni}$ atoms (inset in Figure 3c), which is much smaller than that of LMO. The same sign of the $\mathrm{Ni}$ and $\mathrm{Mn} \mathrm{L}_{2}$ edges indicates that the net spin moments of both layers are aligned parallel, as expected for a $\mathrm{Ni}^{2+}-\mathrm{Mn}^{4+}$ ferromagnetic configuration. No signature of magnetic dichroism is observed for the sharp-interface sample or the LNO films.

Resonant soft X-ray reflectivity measurements allow us to reconstruct the magnetic depth profile across the LMO layers. ${ }^{42}$ These measurements were performed at the SEXTANTS beamline of the SOLEIL synchrotron. ${ }^{43}$ Figure 4 displays the reflectivity curves acquired with circularly left $(\mathrm{CL})$ polarized light in $+0.1 \mathrm{~T}$ and $-0.1 \mathrm{~T}$ for a set of bilayers $N=M=7$ u.c. at $\mathrm{Mn}_{3}$ edge at $30 \mathrm{~K}$ after cooling in $0.05 \mathrm{~T}$. At the strongly resonant $\mathrm{Mn} \mathrm{L}_{3}$ edge, the difference between the reflectivity curves of the two bilayers is striking as the oscillation period seems doubled in the LMO-on-LNO sample. This is another clear indication of the interface quality as a rough interface produces oscillations related to the thickness of the entire stack, whereas a smooth interface creates interferences between the two individual components' thicknesses, which are very close, and therefore, the $q$ space oscillations almost superimpose. Indeed, fits carried out using DYNA ${ }^{44}$ give a $(7.5 \pm 1.2) \AA$ roughness at the intermixed LNO-on-LMO interface, and (2.7 $\pm 0.7) \AA$ for the smooth one, in very good agreement with the TEM results (Figure 1$)$. The asymmetry ratio $\left(\mathrm{CL}^{+}-\mathrm{CL}^{-}\right) /$ $\left(\mathrm{CL}^{+}+\mathrm{CL}^{-}\right)$at $\mathrm{Mn} \mathrm{L}_{3}$-edge are shown in the insets of Figure $4 \mathrm{a}$ and $\mathrm{c}$, along with their fits, and the reconstructed LMO magnetic profiles are plotted in panel e. These reveal that the magnetization of the LMO layer in the rough interface configuration is maximum at the interface with LNO and diminishes away from it. For the sharp interface, the depth profile shows maximum LMO magnetization in the inner layers, and it is depressed in the vicinity of both interfaces, i.e. vacuum and LNO. Additionally, the overall magnitude of the magnetization is strongly reduced compared to the rough bilayer and appears to be negligible at the interface with LNO (bottom). From polarized X-ray reflectivity measurements at the $\mathrm{Ni} \mathrm{L}_{2}$ edge (Figure $4 \mathrm{~b}, \mathrm{~d}$ ), one can clearly see an asymmetry between the two polarizations only for the rough bilayer LNOon-LMO. In this case, the proximity-induced magnetism is found from the fits to extend over $(3.5 \pm 1.5) \AA$ from the interface. This result is consistent with the intermixing effect expected to induce ferromagnetic superexchange, which perturbs the antiferromagnetic order at the atomic layer scale. No magnetic component is induced in the LNO layer for the smooth LMO-on-LNO interface (Figure 4d), confirming that in this case $\mathrm{Ni}-\mathrm{Mn}$ bonds are not dominant.

At this stage, the enhanced magnetization of the rough interface bilayers can already be attributed to several contributions. First, ferromagnetic coupling between $\mathrm{Ni}^{2+}-$ $\mathrm{Mn}^{4+}$ cations resulting from interfacial charge transfer. Second, a capping effect, as previously discussed for LNO in the context of transport properties, also boosting magnetization by avoiding the deterioration of the upper LMO monolayers. Indeed, 

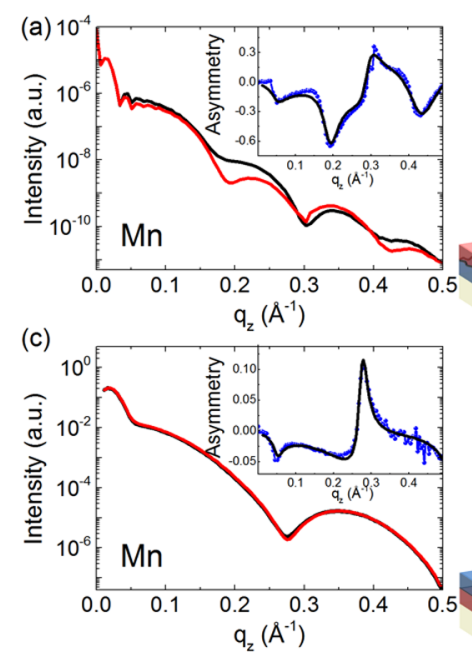

(b)

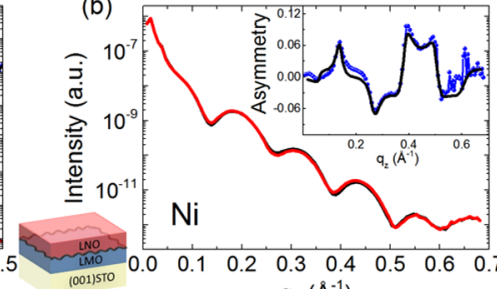
$\mathrm{q}_{\mathrm{z}}\left(\AA^{-1}\right)$

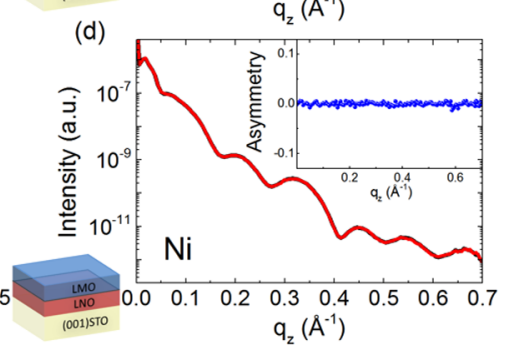

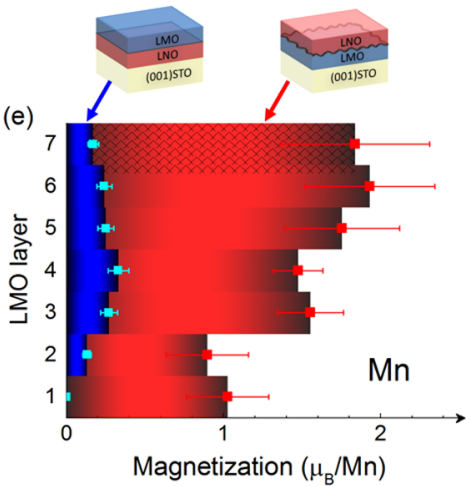

Magnetization $\left(\mu_{\mathrm{B}} / \mathrm{Mn}\right)$

Figure 4. X-ray reflectivity curves acquired in $-0.1 \mathrm{~T}$ (black) and $+0.1 \mathrm{~T}$ (red) circular left polarized light at the $\mathrm{Mn}$ (and $\mathrm{Ni}$ ) $\mathrm{L}_{3}$ edge at $30 \mathrm{~K}$ and $0.05 \mathrm{~T}$ for $(\mathrm{a},(\mathrm{b}))$ a rough interface $(7 \mathrm{LNO} / 7 \mathrm{LMO})_{1} / /(001) \mathrm{STO}$ and $(\mathrm{c},(\mathrm{d}))$ an abrupt interface $(7 \mathrm{LMO} / 7 \mathrm{LNO})_{1} / /(001)$ STO bilayer. Insets: Corresponding asymmetry ratio curves (blue) and best fits to the data (black). (e) Plot of the depth magnetic profile of the LMO layer determined from the fittings for the rough (red) and abrupt (blue) interface bilayer configurations. Square symbols indicate the extracted average magnetization value as a function of thickness, and the hatched area indicates the Ni-Mn intermixed region as inferred from the fit in (a).

capping the LMO films with LAO also results in a slight increase of total magnetization (SI). Moreover, a small magnetic contribution of the LNO layers is also present. It should be noticed that hole-doping of the whole managanite layer plays a minor role at most to such enhanced magnetization since no corresponding increase of conductivity has been observed for this bilayer type. Interestingly, the resistivity of the intermixed LNO-on-LMO bilayer could be expected to be higher than that of the bare LNO film. The fact that this is not the case suggests that the expected decrease of conductivity due to intermixing might be compensated by the increase of conductivity of the interfacial manganite layers.

For the sharp LMO-on-LNO interface bilayer, both XMCD hysteresis loops and extracted magnetic profile point to a canted antiferromagnetic order for LMO when grown on LNO and coherently strained to STO. Further evidence for the stabilization of such an antiferromagnetic phase is obtained from X-ray magnetic linear dichroism (XMLD) measurements presented in SI. Moreover, a systematic study of a series of sharp-interface LMO-on-LNO bilayers in which the thickness of the top LMO layer was progressively increased while keeping LNO layer constant at $N=7$ u.c. showed that the total magnetization of the heterostructures rises upon increasing the LMO thickness and approaches the magnetic moment of the bare LMO thin films (all structures being strained to the STO lattice). These results were confirmed both by SQUID magnetometry and XMCD measurements (SI). Consequently, the canted-antiferromagnetic phase of LMO when grown on LNO is the result of an interface-driven phenomenon.

The sharpness of the LMO-on-LNO interface also appears to be key for the development of such a magnetic structure. To test this important point, we realized LMO-on-LNO bilayers with artificially generated intermixed interfaces; this is done by depositing at the interface submonolayers of LMO and LNO mimicking an intermixed region. Doing so, one is turning the sharp interface bilayers in a rough one. As shown in the SI, the measured magnetic moment is indeed enhanced as compared to the equivalent abrupt-interface samples demonstrating the role of the sharp interface in achieving the cantedantiferromagnetic phase in LMO films.
As previously mentioned, the magnetic ground state of bulk LMO exhibits a canted-antiferromagnetic order, which can be easily pushed into a ferromagnetic state through epitaxial strain. $^{16,17}$ In addition to strain, octahedral connectivity requirements across the interface between different perovskite materials can be another source of interfacial lattice reconstructions. ${ }^{45}$ Indeed, dissimilar electronic properties have been reported for Sr-doped LMO thin films grown on substrates with similar lattice parameters but different symmetry. ${ }^{46,47}$ Bulk orthorhombic LMO is characterized by an $a^{-} a^{-} c^{+}$tilt pattern (in Glazer notation) with $\mathrm{Mn}-\mathrm{O}-\mathrm{Mn}$ bond angle $\theta_{\mathrm{Mn}-\mathrm{O}-\mathrm{Mn}} \approx 155^{\circ}$, whereas rotations are absent in cubic STO $\left(a^{0} a^{0} a^{0}, \theta_{\mathrm{Ti}-\mathrm{O}-\mathrm{Ti}} \approx 180^{\circ}\right.$ at room temperature). Consequently, in addition to lattice parameter mismatch, modifications of the bulk LMO rotation pattern are indeed expected to occur in response to the constraints imposed by the substrate and/or the dissimilar neighboring layer, with important consequences for the electronic properties due to the sensitivity of the LMO magnetic order to cooperative Jahn-Teller distortions and orbital ordering. For example, an evolution from enhanced to quenched magnetic moment has previously been observed in LMO/STO superlattices as oxygen octahedral rotations are modified from low to high angles. ${ }^{48}$ LNO films grown on STO exhibit a rotation pattern $a^{-} a^{-} c^{-}$and average $\theta_{\mathrm{Ni}-\mathrm{O}-\mathrm{Ni}} \approx 164^{\circ} .{ }^{49}$ In our system, inserting a LNO film between the LMO layer and the STO substrate stabilizes a canted antiferromagnetic state in LMO despite keeping inplane the STO lattice parameter. Whether the induced antiferromagnetic structure presents the same pattern as bulk LMO remains an open question. As observed, the sharpness of the interface is likely to help for the propagation of the interfacial couplings. It should also be noted that the typical length scales for the oxygen octahedral reconstructions induced at the interface, and consequent modification of electronic properties extend over $2-10$ u.c., ${ }^{4750-52}$ in agreement with the thickness range where the canted antiferromagnetism of the LMO is observed to occur in this work when grown on LNO// (001)STO (SI).

In conclusion, we have shown that interfacial intermixing at a single monolayer scale can have strong consequences on the 
resulting properties of oxide heterostructures. Focusing on the LMO/LNO system, we report that the stabilized magnetic structure of LMO is strongly related to the interfacial reconstructions, which depend sensitively on the exact interface morphology. In particular, we report here that a single monolayer change in intermixing is able to significantly modify the magnetic state of the manganite, from canted-antiferromagnetic to ferromagnetic, and the induced magnetic moment in interfacial LNO layers. This underlines that in order to tailor specific properties in an oxide superlattice, the choice of materials and periodicity are not the only important parameters. The exact local interface geometry can also greatly influence charge transfer and magnetic exchange. Further progress in deposition and materials control at the atomic scale should make this interface parameter an important new tool to better control artificial functionalities through interfaces.

\section{ASSOCIATED CONTENT}

\section{S Supporting Information}

The Supporting Information is available free of charge on the ACS Publications website at DOI: 10.1021/acs.nanolett.5b02720.

Structural characterization of bilayers, additional transport, magnetic, transmission electron microscopy, X-ray absorption spectroscopy, and X-ray magnetic linear dichroism measurements (PDF)

\section{AUTHOR INFORMATION}

\section{Corresponding Author}

*E-mail: marta.gibert@unige.ch.

\section{Present Addresses}

(A.T.-P.) Facultad de Ciencias Químicas, Complutense University of Madrid, 28040 Madrid, Spain.

(P.Z.) University College London, London Centre for Nanotechnology, London, WC1H 0AH, U.K.

\section{Notes}

The authors declare no competing financial interest.

\section{ACKNOWLEDGMENTS}

We thank M. Lopes and S. Muller for technical support. We also like to thank O. Peil, E. Bousquet, and D. Muller for valuable discussions. This work was supported by the Swiss National Science Foundation through Division II. The research leading to these results has received funding from the European Research Council under the European Union's Seventh Framework Program (FP7/2007-2013)/ERC Grant Agreement no. 319286 (Q-MAC). The X-ray absorption measurements were (partially) performed on the EPFL/PSI X-Treme beamline at the Swiss Light Source, Paul Scherrer Institut, Villigen, Switzerland. M.G. acknowledge the funding from University of Geneva through the "Boursière d'excellence" program. A.T.-P. is also grateful for funding from the Spanish Ministry of Education and Science through the MEC postdoctoral fellowship EX2009-0156.

\section{REFERENCES}

(1) Zubko, P.; Gariglio, S.; Gabay, M.; Ghosez, P.; Triscone, J.-M. Annu. Rev. Condens. Matter Phys. 2011, 2, 141.

(2) Hwang, H. Y.; Iwasa, Y.; Kawasaki, M.; Keimer, B.; Nagaosa, N.; Tokura, Y. Nat. Mater. 2012, 11, 103.

(3) Ohtomo, A.; Muller, D. A.; Grazul, J. L.; Hwang, H. Y. Nature 2002, 419, 378 .
(4) Koida, T.; Lippmaa, M.; Fukumura, T.; Itaka, K.; Matsumoto, Y.; Kawasaki, M.; Koinuma, H. Phys. Rev. B: Condens. Matter Mater. Phys. 2002, 66, 144418.

(5) Reyren, N.; Thiel, S.; Caviglia, A. D.; Kourkoutis, L. F.; Hammerl, G.; Richter, C.; Schneider, C. W.; Kopp, T.; Ruetschi, A.-S.; Jaccard, D.; Gabay, M.; Muller, D. A.; Triscone, J.-M.; Mannhart, J. Science 2007, 317, 1196

(6) Boris, A. V.; Matiks, Y.; Benckiser, E.; Frano, A.; Popovich, P.; Hinkov, V.; Wochner, P.; Castro-Colin, M.; Detemple, E.; Malik, V. K.; Bernhard, C.; Prokscha, T.; Suter, A.; Salman, Z.; Morenzoni, E.; Cristiani, G.; Habermeier, H.-U.; Keimer, B. Science 2011, 332, 937.

(7) Schlom, D. G.; Chen, L. Q.; Pan, X. Q.; Schmehl, A.; Zurbuchen, M. A. J. Am. Ceram. Soc. 2008, 91, 2429.

(8) Nakagawa, N.; Hwang, H. Y.; Muller, D. A. Nat. Mater. 2006, 5, 204.

(9) Kourkoutis, L. F.; Muller, D. A.; Hotta, Y.; Hwang, H. Y. Appl. Phys. Lett. 2007, 91, 163101.

(10) May, S. J.; Shah, A. B.; Velthuis, S.; Fitzsimmons, M. R.; Zuo, J. M.; Zhai, X.; Eckstein, J. N.; Bader, S. D.; Bhattacharya, A. Phys. Rev. B: Condens. Matter Mater. Phys. 2008, 77, 174409.

(11) Wollan, E. O.; Koehler, W. C. Phys. Rev. 1955, 100, 545.

(12) Topfer, J.; Goodenough, J. B. J. Solid State Chem. 1997, 130, 117.

(13) Skumryev, V.; Ott, F.; Coey, J. M. D.; Anane, A.; Renard, J. P.; Pinsard-Gaudart, L.; Revcolevschi, A. Eur. Phys. J. B 1999, 11, 401.

(14) Ritter, C.; Ibarra, M. R.; De Teresa, J. M.; Algarabel, P. A.; Marquina, C.; Blasco, J.; García, J.; Oseroff, S.; Cheong, S. W. Phys. Rev. B: Condens. Matter Mater. Phys. 1997, 56, 8902.

(15) Gupta, A.; McGuire, T. R.; Duncombe, P. R.; Rupp, M.; Sun, J. Z.; Gallagher, W. J.; Xiao, G. Appl. Phys. Lett. 1995, 67, 3494.

(16) Dong, S.; Yu, R.; Yunoki, S.; Alvarez, G.; Liu, J. M.; Dagotto, E. Phys. Rev. B: Condens. Matter Mater. Phys. 2008, 78, 201102.

(17) Lee, J. H.; Delaney, K. T.; Bousquet, E.; Spaldin, N. A.; Rabe, K. M. Phys. Rev. B: Condens. Matter Mater. Phys. 2013, 88, 174426.

(18) Hou, Y. S.; Xiang, H. J.; Gong, X. G. Phys. Rev. B: Condens. Matter Mater. Phys. 2014, 89, 064415.

(19) Wang, X. R.; Li, C. J.; Lü, W. M.; Paudel, T. R.; Leusink, D. P.; Hoek, M.; Poccia, N.; Vailionis, A.; Venkatesan, T.; Coey, J. M. D.; Tsymbal, E. Y.; Ariando; Hilgenkamp, H. Science 2015, 349, 716.

(20) Rajeev, K. P.; Shivashankar, G. V.; Raychaudhuri, A. K. Solid State Commun. 1991, 79, 591.

(21) García-Munoz, J. L.; Rodríguez-Carvajal, J.; Lacorre, P.; Torrance, J. B. Phys. Rev. B: Condens. Matter Mater. Phys. 1992, 46, 4414.

(22) Sreedhar, K.; Honig, J. M.; Darwin, M.; McElfresh, M.; Shand, P. M.; Xu, J.; Crooker, B. C.; Spalek, J. Phys. Rev. B: Condens. Matter Mater. Phys. 1992, 46, 6382.

(23) Scherwitzl, R.; Zubko, P.; Lichtensteiger, C.; Triscone, J. M. Appl. Phys. Lett. 2009, 95, 222114.

(24) Son, J.; Moetakef, P.; LeBeau, J. M.; Ouellette, D.; Balents, L.; Allen, S. J.; Stemmer, S. Appl. Phys. Lett. 2010, 96, 062114.

(25) Gibert, M.; Zubko, P.; Scherwitzl, R.; Íñiguez, J.; Triscone, J.-M. Nat. Mater. 2012, 11, 195.

(26) Rojas Sánchez, J. C.; Nelson-Cheeseman, B.; Granada, M.; Arenholz, E.; Steren, L. B. Phys. Rev. B: Condens. Matter Mater. Phys. 2012, 85, 094427.

(27) Hoffman, J.; Tung, I. C.; Nelson-Cheeseman, B. B.; Liu, M.; Freeland, J. W.; Bhattacharya, A. Phys. Rev. B: Condens. Matter Mater. Phys. 2013, 88, 144411.

(28) Piamonteze, C.; Gibert, M.; Heidler, J.; Dreiser, J.; Rusponi, S.; Brune, H.; Triscone, J. M.; Nolting, F.; Staub, U. Phys. Rev. B: Condens. Matter Mater. Phys. 2015, 92, 014426.

(29) Di Pietro, P.; Hoffman, J.; Bhattacharya, A.; Lupi, S.; Perucchi, A. Phys. Rev. Lett. 2015, 114, 156801.

(30) Paulsen, J. M.; Thomas, C. L.; Dahn, J. R. J. Electrochem. Soc. 2000, 147, 861.

(31) Bhattacharya, A.; May, S. J. Annu. Rev. Mater. Res. 2014, 44, 65.

(32) Goodenough, J. Magnetism and Chemical Bond; Interscience (Wiley): New York, 1963. 
(33) González-Jiménez, I. N.; Torres-Pardo, A.; Sánchez-Peláez, A. E.; Gutiérrez, A.; García-Hernández, M.; González-Calbet, J. M.; Parras, M.; Varela, Á. Chem. Mater. 2014, 26, 2256.

(34) Abbate, M.; de Groot, F. M. F.; Fuggle, J. C.; Fujimori, A.; Strebel, O.; Lopez, F.; Domke, M.; Kaindl, G.; Sawatzky, G. A.; Takano, M.; Takeda, Y.; Eisaki, H.; Uchida, S. Phys. Rev. B: Condens. Matter Mater. Phys. 1992, 46, 4511.

(35) Thole, B.; van der Laan, G. Phys. Rev. B: Condens. Matter Mater. Phys. 1988, 38, 3158.

(36) Mundy, J. A.; Hikita, Y.; Hidaka, T.; Yajima, T.; Higuchi, T.; Hwang, H. Y.; Muller, D. A.; Kourkoutis, L. F. Nat. Commun. 2014, 5, 3464.

(37) Middey, S.; Rivero, P.; Meyers, D.; Kareev, M.; Liu, X.; Cao, Y.; Freeland, J. W.; Barraza-Lopez, S.; Chakhalian, J. Sci. Rep. 2014, 4, 6819.

(38) Kumah, D. P.; Disa, A. S.; Ngai, J. H.; Chen, H.; Malashevich, A.; Reiner, J. W.; Ismail-Beigi, S.; Walker, F.-J.; Ahn, C. H. Adv. Mater. 2014, 26, 1935.

(39) Ferre, J.; Gehring, G. A. Rep. Prog. Phys. 1984, 47, 513.

(40) Piamonteze, C.; Flechsig, U.; Rusponi, S.; Dreiser, J.; Heidler, J.; Schmidt, M.; Wetter, R.; Calvi, M.; Schmidt, T.; Pruchova, H.; Krempasky, J.; Quitmann, C.; Brune, H.; Nolting, F. J. Synchrotron Radiat. 2012, 19, 661.

(41) Coey, J. M. D.; Viret, M.; von Molnar, S. Adv. Phys. 1999, 48, 167.

(42) Tonnerre, J.-M.; Jaouen, N.; Bontempi, E.; Carbone, D.; Babonneau, D.; De Santis, M.; Tolentino, H. C. N.; Grenier, S.; Garaudee, S.; Staub, U. J. Phys. Conf Ser. 2010, 211, 012015.

(43) Jaouen, N.; Tonnerre, J. M.; Kapoujian, G.; Taunier, P.; Roux, J. P.; Raoux, D.; Sirotti, F. J. Synchrotron Radiat. 2004, 11, 353.

(44) Elzo, M.; Jal, E.; Bunau, O.; Grenier, S.; Joly, Y.; Ramos, A. Y.; Tolentino, H. C. N.; Tonnerre, J. M.; Jaouen, N. J. Magn. Magn. Mater. 2012, 324, 105.

(45) Rondinelli, J. M.; May, S. J.; Freeland, J. W. MRS Bull. 2012, 37, 261.

(46) Tebano, A.; Aruta, C.; Sanna, S.; Medaglia, P. G.; Balestrino, G.; Sidorenko, A. A.; De Renzi, R.; Ghiringhelli, G.; Braicovich, L.; Bisogni, V.; Brookes, N. B. Phys. Rev. Lett. 2008, 100, 137401.

(47) Moon, E. J.; Balachandran, P. V.; Kirby, B. J.; Keavney, D. J.; Sichel-Tissot, R. J.; Schlepütz, C. M.; Karapetrova, E.; Cheng, X. M.; Rondinelli, J. M.; May, S. J. Nano Lett. 2014, 14, 2509.

(48) Zhai, X.; Cheng, L.; Liu, Y.; Schlepütz, C. M.; Dong, S.; Li, H.; Zhang, X.; Chu, S.; Zheng, L.; Zhang, J.; Zhao, A.; Hong, H.; Bhattacharya, A.; Eckstein, J. N.; Zeng, C. Nat. Commun. 2014, 5, 4283.

(49) May, S. J.; Kim, J. W.; Rondinelli, J. M.; Karapetrova, E.; Spaldin, N. A.; Bhattacharya, A.; Ryan, P. J. Phys. Rev. B: Condens. Matter Mater. Phys. 2010, 82, 014110.

(50) Boschker, H.; Kautz, J.; Houwman, E. P.; Siemons, W.; Blank, D. H. A.; Huijben, M.; Koster, G.; Vailionis, A.; Rijnders, G. Phys. Rev. Lett. 2012, 109, 157207.

(51) Hwang, J.; Son, J.; Zhang, J. Y.; Janotti, A.; Van de Walle, C. G.; Stemmer, S. Phys. Rev. B: Condens. Matter Mater. Phys. 2013, 87, 060101.

(52) Kan, D.; Aso, R.; Kurata, H.; Shimakawa, Y. APL Mater. 2015, 3, 062302. 